\title{
New Aggregation Operator for Triangular Fuzzy Numbers based on the Geometric Means of the Slopes of the L- and R- Membership Functions
}

\author{
Manju Pandey \\ Research Scholar \\ Computer Science \& Engg \\ MANIT, Bhopal, India
}

\author{
Dr. Nilay Khare \\ Professor \& Head \\ Computer Science \& Engg \\ MANIT, Bhopal, India
}

\author{
Dr. S.C. Shrivastava \\ Former Professor \& Head \\ Computer Science \& Engg \\ and Electronics \& \\ Communication Engg \\ MANIT, Bhopal, India
}

\begin{abstract}
In recent work authors have proposed four new aggregation operators for triangular and trapezoidal fuzzy numbers based on means of apex angles [1][2][3][4]. Subsequently authors have proposed [5] a new aggregation operator for TFNs based on the arithmetic mean of slopes of the L- and R- membership lines. In this paper the work is extended and a new aggregation operator for TFNs is proposed in which the L- and R- membership function lines of the aggregate TFN have slopes which are the geometric means of the corresponding L-and R-slopes of the individual TFNs. Computation of the aggregate is demonstrated with a numerical example. Corresponding arithmetic and geometric aggregates as well as results from the recent work of the authors on TFN aggregates have also been computed.
\end{abstract}

\section{General Terms}

Fuzzy Aggregation, Aggregation Operators, Fuzzy Logic, Fuzzy Sets, Fuzzy Numbers, Fuzzy Arithmetic, Fuzzy Mathematics

\section{Keywords}

LR Fuzzy Number, Triangular Fuzzy Number (TFN), Trapezoidal Fuzzy Number ( $\mathrm{TrFN}$ ), Apex Angle, Slope, L- and R- Membership Functions, Aggregation Operator, Arithmetic and Geometric Mean

\section{INTRODUCTION}

Many different types of fuzzy numbers are defined in the literature dealing with fuzzy logic and applications. In this paper only one class of fuzzy numbers i.e., Triangular Fuzzy Numbers (TFNs) which are a special class of LR fuzzy numbers are treated.

\subsection{Triangular Fuzzy Numbers}

TFNs are extensively used in fuzzy applications owing to their simplicity. TFNs are used in fuzzy applications where uncertainty exists on both sides of a value or parameter [6]. TFNs are characterized by an ordered triplet of real numbers $\langle 1, \mathrm{~m}, \mathrm{u}\rangle$. Figure depicts a TFN $(1, \mathrm{~m}, \mathrm{u})$ with values $\mathrm{v}$ on $\mathrm{x}$-axis and membership or grade $\mu$ along y-axis. The L- and R- apex angles are shown with arcs having one and two dashes respectively. Vertices of the triangle are at $(1,0),(m, 1) \&(u, 0)$ moving clockwise. Line between $(1,0)$ and $(m, 1)$ and between $(\mathrm{m}, 1)$ and $(\mathrm{u}, 0)$ are the membership functions for the values in the intervals $[1, \mathrm{~m}]$ and $[\mathrm{m}, \mathrm{u}]$ respectively. Membership function of the TFN is the piecewise continuous linear function represented by the lines $\mathrm{L}$ and $\mathrm{R}$ respectively. Intuitive meaning of such TFN is that the fuzzy number is approximately $m$ [7]. The value of the fuzzy number varies in the range 1 and $u$. The possibility or membership grade of the number being a specified value $\mathrm{v}$ between $\mathrm{l}$ and $\mathrm{u}, \mathrm{v} \in[1, \mathrm{u}]$ is represented by the ordinate of the projection of $\mathrm{v}$ on $\mathrm{L}$ or $\mathrm{R}$ accordingly as $\mathrm{l} \leq \mathrm{v} \leq \mathrm{m}$ or $\mathrm{m} \leq$ $\mathrm{v} \leq \mathrm{u} .[1, \mathrm{~m}]$ is the range or interval which depicts or represents the possibilities of the number being a specific value less than $\mathrm{m}$ and $[\mathrm{m}, \mathrm{u}]$ is the interval which depicts the possibilities of the number being a specific value greater than $\mathrm{m}$. Possibility takes on the maximum value of 1 at $\mathrm{m}$ and reduces with increasing distance on either side (left / right) of $\mathrm{m}$, becoming zero beyond 1 at the left and $m$ at the right respectively.

Mathematically, a TFN is defined as follows. Let $l, m, u \in \mathbf{R}, l<$ $m<u$. The fuzzy number $t: \mathbf{R} \rightarrow[0,1]$ denoted by

$t= \begin{cases}0, & \text { if } x<l \\ \frac{x-l}{m-l}, & \text { if } l \leq x \leq m \\ \frac{u-x}{u-m}, & \text { if } m \leq x \leq u \\ 0, & \text { if } x>u\end{cases}$

is called a triangular fuzzy number [6][7][8][9].

\section{Fuzzy Aggregation}

Aggregation operations on fuzzy numbers are operations by which several fuzzy numbers are combined to produce a single fuzzy number [10]. An excellent account of Mathematical Aggregation Operators is given in [11] 


\subsection{Arithmetic Mean}

The arithmetic mean aggregation operator [10][12] defined on $n$ TFNs $\left\langle\mathrm{l}_{1}, \mathrm{~m}_{1}, \mathrm{u}_{1}\right\rangle,\left\langle\mathrm{l}_{2}, \mathrm{~m}_{2}, \mathrm{u}_{2}>, \ldots,<\mathrm{l}_{\mathrm{i}}, \mathrm{m}_{\mathrm{i}}, \mathrm{u}_{\mathrm{i}}>, \ldots,<\mathrm{l}_{\mathrm{n}}, \mathrm{m}_{\mathrm{n}}, \mathrm{u}_{\mathrm{n}}\right\rangle$ produces the result $\langle\bar{l}, \bar{m}, \bar{u},>$ where

$$
\bar{l}=\frac{1}{n} \sum_{1}^{n} l_{i}, \bar{m}=\frac{1}{n} \sum_{1}^{n} m_{i}, \text { and } \bar{u}=\frac{1}{n} \sum_{1}^{n} u_{i}
$$

\subsection{Geometric Mean}

The geometric mean aggregation operator [10][13] defined on $n$ TFNs $\left\langle\mathrm{l}_{1}, \mathrm{~m}_{1}, \mathrm{u}_{1}>,<\mathrm{l}_{2}, \mathrm{~m}_{2}, \mathrm{u}_{2}>, \ldots,<\mathrm{l}_{\mathrm{i}}, \mathrm{m}_{\mathrm{i}}, \mathrm{u}_{\mathrm{i}}>, \ldots,<\mathrm{l}_{\mathrm{n}}, \mathrm{m}_{\mathrm{n}}, \mathrm{u}_{\mathrm{n}}>\right.$ produces the result $\langle\bar{l}, \bar{m}, \bar{u},>$ where

$$
\bar{l}=\left(\prod_{1}^{n} l_{i}\right)^{\frac{1}{n}}, \bar{m}=\left(\prod_{1}^{n} m_{i}\right)^{\frac{1}{n}} \text {, and } \bar{u}=\left(\prod_{1}^{n} u_{i}\right)^{\frac{1}{n}}
$$

Other aggregation operators have also been defined in literature. For examples see [13][14][15][16].

\subsection{Applications of Aggregation}

The combination/aggregation/fusion of information from different sources is at the core of knowledge based systems. Applications include decision making, subjective quality evaluation, information integration, multi-sensor data fusion, image processing, pattern recognition, computational intelligence etc. An application of aggregation operators in fuzzy multicriteria decision making is discussed in [12][13]. Another application in sensor data fusion is discussed in [15].

\section{Proposed Aggregation Operator}

In this paper a new aggregation operator for TFNs based on arithmetic means of the slopes is proposed. The L- and Rmembership functions have been treated independently. The operator is described in the next section. Computation of the aggregate is demonstrated with a numerical example. Corresponding arithmetic and geometric aggregates as well as results from the recent work of the authors on TFN aggregates have also been computed

Consider the TFN shown in Fig. 1. The most likely value of this TFN is $\mathrm{m}$ where the possibility $\mu=1$. The slope of L- membership line is $\tan (\mathrm{Lmlp})$ while the slope of the R-membership line is $\tan (\operatorname{Lmux})=-\tan ($ Lpum $)$

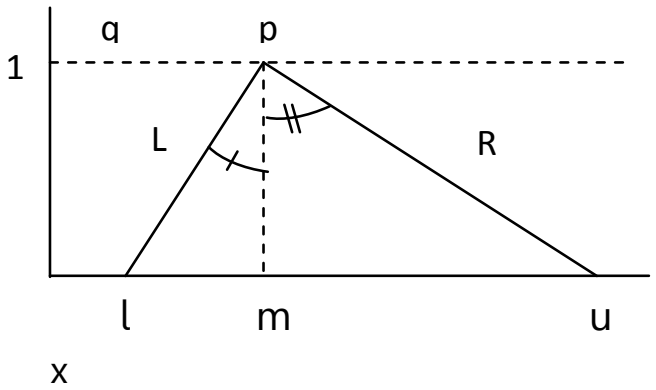

Fig.1: Triangular Fuzzy Number
Computing the geometric mean of the L-slopes over $n$ TFNs we have

$$
\left(\prod_{1}^{\mathrm{n}} \tan (\operatorname{Lmlp})_{i}\right)^{\frac{1}{\mathrm{n}}}=\left(\prod_{1}^{\mathrm{n}} \frac{1}{\left(m_{i}-l_{i}\right)}\right)^{\frac{1}{\mathrm{n}}}
$$

This represents the slope of the resultant aggregate left line $\mathrm{L}$

Similarly, it can be shown that the slope of the resultant fuzzy aggregate right line $\overline{\mathrm{R}}$ is $-\left(\prod_{1}^{\mathrm{n}} \frac{1}{\left(u_{i}-m_{i}\right)}\right)^{\frac{1}{\mathrm{n}}}$

Under identical treatment, it can be shown that $\overline{\mathrm{m}}=\left(\prod_{1}^{\mathrm{n}} \mathrm{m}_{\mathrm{i}}\right)^{\frac{1}{\mathrm{n}}}$.

Subsequently it can be shown that

$\overline{1}=\left(\prod_{1}^{\mathrm{n}} \mathrm{m}_{\mathrm{i}}\right)^{\frac{1}{\mathrm{n}}}-\frac{1}{\left(\prod_{1}^{\mathrm{n}} \frac{1}{\left(m_{i}-l_{i}\right)}\right)^{\frac{1}{\mathrm{n}}}}$, and

$\overline{\mathrm{u}}=\left(\prod_{1}^{\mathrm{n}} \mathrm{m}_{\mathrm{i}}\right)^{\frac{1}{\mathrm{n}}}+\frac{1}{\left(\prod_{1}^{\mathrm{n}} \frac{1}{\left(u_{i}-m_{i}\right)}\right)^{\frac{1}{\mathrm{n}}}}$

\section{Numerical Example}

Consider the two triangular fuzzy numbers $\langle\mathbf{1 , 1 . 5 , 3}\rangle$ and <5,6.5,9> aggregate TFN is computed as $\bar{m}=\overline{\mathrm{m}}=\sqrt{1.5 \times 6.5}=3.1224$;

$$
\bar{l}=3.1224-\frac{1}{\left(\frac{1}{(1.5-1)} \times \frac{1}{(6.5-5)}\right)^{1 / 2}}=2.2564 \text {; }
$$

Similarly, $\bar{u}$ can be computed as 5.059 .

We have the aggregate as $<\mathbf{2 . 2 5 6 4 , 3 . 1 2 2 4 , 5 . 0 5 9 >}$. The arithmetic mean aggregate is $\langle\mathbf{3 , 4 , 6}\rangle$ and the geometric mean aggregate is $\langle\mathbf{2 . 2 4 , 3 . 1 2 , 5 . 1 9}\rangle$ respectively. We have the aggregate as $\langle\mathbf{3 . 1 1 7 , 4 , 5 . 9 0}\rangle$ considering arithmetic means of apex angles and $\langle\mathbf{2 . 3 2 1 9 , 3 . 1 2 2 4 , 4 . 2 0 \rangle}$ considering geometric mean of apex angles. We have the aggregate as $\mathbf{3} \mathbf{3 . 2 5 , 4 , 5 . 8 7 5}\rangle$ considering arithmetic mean of slopes. 


\section{Conclusions}

In this paper we have defined a new aggregate of triangular fuzzy numbers based on the geometric mean of the slopes. The L- and R- membership lines and their slopes have been treated independently. A numerical example has been worked out. The aggregate is the resultant piecewise continuous membership function line whose L- and R- slopes are the geometric means of the L- and R- slopes of the individual TFNs. The suitability of the aggregation operator proposed in this paper in different fuzzy logic applications involving fuzzy number aggregation remains to be explored.

\section{Acknowledgements}

Manju Pandey gratefully acknowledges Shri Harendra Bikrol, Associate Professor, and Shri Mithilesh Atulkar, Associate Professor and Head of Computer Applications Department at National Institute of Technology (NIT), Raipur, Chhattisgarh, India, for their constant help and support. She is also thankful to Dr. Shrish Verma, Head of Electronics and Information Technology Departments and Central Computer Centre at NIT Raipur for his encouragement and support.

\section{References}

[1] Manju Pandey, Nilay Khare, and S.C. Shrivastava, "New Aggregation Operator for Triangular Fuzzy Numbers based on the Arithmetic Means of the L- and R- Apex Angles", Submitted for Publication (2012)

[2] Manju Pandey, Nilay Khare, and S.C. Shrivastava, "New Aggregation Operator for Triangular Fuzzy Numbers based on the Geometric Means of the L- and R- Apex Angles", Submitted for Publication (2012)

[3] Manju Pandey, Nilay Khare, and S.C. Shrivastava, "New Aggregation Operator for Trapezoidal Fuzzy Numbers based on the Arithmetic Means of the Left and Right Apex Angles", Submitted for Publication (2012)

[4] Manju Pandey, Nilay Khare, and S.C. Shrivastava, "New Aggregation Operator for Trapezoidal Fuzzy Numbers based on the Geometric Means of the Left and Right Apex Angles", Submitted for Publication (2012)

[5] Manju Pandey, Nilay Khare, and S.C. Shrivastava, "New Aggregation Operator for Triangular Fuzzy Numbers based on the Arithmetic Means of the Slopes of the L- and R- Membership Functions" Submitted for Publication (2012)

[6] A.K. Verma, A.Srividya and R. S Prabhu Gaonkar, Fuzzy-Reliability Engineering Concepts and Applications, Narosa Publishing House Pvt. Limited, 2007

[7] Hung T, Nguyen, Elbert A. Walker, A First Course in Fuzzy Logic, Third Edition, Chapman \& Hall/CRC, Taylor and Francis Group, 2006
[8] Michael Haans, Applied Fuzzy Arithmetic - An Introduction with Engineering Applications, SpringerVerlag, Berlin Heidelberg, 2005

[9] Kauffman, A. and Gupta, M.M., Introduction to Fuzzy Arithmetic - Theory and Applications, Van Nostrand Reinhold Company, New York, 1985

[10] George J. Klir and Tina A. Folger, Fuzzy Sets, Uncertainty, and Information, Prentice-Hall of India Pvt. Ltd., New Delhi, 1988

[11] Marcin Detyniecki, Mathematical Aggregation Operators and their Application to Video Querying, $\mathrm{PhD}$ Thesis in Artificial Intelligence Specialty, University of Paris, (C) 2000 Detyniecki

[12] Mehdi Fasangarhi, Farzad Habibipour Roudsari, "The Fuzzy Evaluation of E-Commerce Customer Satisfaction", World Applied Sciences Journal 4 (2): 164-168, IDOSI Publications 2008

[13] Oliver Meixner, "Fuzzy AHP Group Decision Analysis and its Application for the Evaluation of Energy Sources", Proceedings of the $10^{\text {th }}$ International Symposium on the Analytic Hierarchy/Network Process Multi-criteria Decision Making, July 29 - August 1 2009, University of Pittsburgh, Pittsburgh, Pennsylvania, USA

[ 14] Bing Yi Kang, Ya Juan Zhang, Xin Yang Deng, Ji Yi Wu, Xiao Hong Sun and Yong Deng, "A New Method of Aggregation of Fuzzy Number Based on the Dempster/Shafer Theory", Chinese Control and Decision Conference, OIEEE 2011

[15] Genevieve Cron, Bernard Dubuisson, "A Weighted Fuzzy Aggregation Method”, CIEEE 1998

[16] Ondrej Pavlacka, Jana Talasova, "Application of the Fuzzy Weighted Average of Fuzzy Numbers in Decision Making Models", Proceedings of EUSFLAT Conference 2007 